\title{
Communicability in multicultural contexts: A study with the International Children's Digital Library
}

\author{
Clarisse Sieckenius de Souza, Robin Fred Laffon, Carla Faria Leitão \\ SERG - Semiotic Engineering Research Group, Depto. de Informática, \\ PUC-Rio, Brazil, \{clarisse, rlaffon, cfaria@inf.puc-rio.br\}
}

\begin{abstract}
This paper presents some contributions of semiotic engineering to the identification of cultural issues involved in the design and evaluation of multicultural systems (i.e. systems designed for users from different cultures). We carried out a communicability evaluation of the International Children's Digital Library. Participants of test sessions had different nationalities and spoke different native languages. We paid special attention to communicability problems stemming from language understanding and language use issues. Our goal was not to make generalizations from our findings, but rather to uncover and understand some of the users' experience with multicultural systems. In addition to this understanding, we have gained relevant insights to inform the cultural adaptation of ICDL to the Brazilian context, and believe that they can be useful in other cultural contexts as well.
\end{abstract}

Keywords: Semiotic engineering, communicability evaluation, cultural issues.

\section{Introduction}

The dissemination of Information and Communication Technology (ICT) has brought about considerable cultural changes. ICT users come from ever more diverse cultural backgrounds, and technology continually fosters new cultural exchange (Castells, 2001). Stimulated by this scenario, the HCI community has set out to investigate design and evaluation methods and techniques that can improve the users' experience when cultural issues are involved. Cultural usability studies propose design strategies centered on both internationalization (to develop sufficiently generic interfaces, suitable for users from different cultures) and localization (to develop customized interfaces for specific cultures) (Aykin, 2005; Dray, 1996; Marcus, 2005). 
These studies have been particularly important for multicultural systems whose users do have different cultural origins, but whose primary purpose does not include cultural exchange. Such is the case of web search systems, for example, where internationalization and localization have brought concrete improvements for the users' experience. However, when it comes to systems whose main purpose is precisely to support social exchange among people from different cultures, internationalization and localization fall short of offering much needed solutions. These systems aim to communicate culture for people from different cultures. Such is the case of websites designed to support travelers' exchange of stories and experience from all over the world. In a context like this, localization is barely useful because it prevents users from experiencing cultural diversity, which is ultimately what they are looking for. And internationalization may arguably be subtracting (for sake of generic solutions) some specifics ('the local flavor') that have a great value for users. A useful metaphor, for sake of introduction, is to say that cultural exchange systems should provide the equivalent of a 'tourist information service'. They should allow users to experience diversity and local values and practices, but at the same time they should prove cultural scaffolds (brief informative descriptions and narratives, tips.) for visitors and newcomers.

This paper presents some preliminary contributions of semiotic engineering (de Souza, 2005), a semiotically-inspired theory of human-computer interaction (HCI), to conceptual design of multicultural systems. These contributions stem from an evaluation study carried out with ICDL - the International Children's Digital Library. ICDL is a specialized public digital library on the Internet, with children's books from throughout the world. An initiative of researchers from the University of Maryland, its mission is "to support the world's children in becoming effective members of the global community - who exhibit tolerance and respect for diverse cultures, languages and ideas" (ICDL, s/d). Among many design challenges, ICDL must support multicultural human-computer interaction.

In semiotic engineering, the main function of computer systems interfaces is to bring designers and users together at interaction time, and to communicate the designers' vision to users through computer-human interaction. This integrative view of a continuous communication process expresses - directly and/or indirectly - the goals and purpose of technology, the choice of interactive strategies and tactics, and in the case of cultural exchange systems, a characterization of different cultures, how they are approached by the designers, and also how users can navigate from one culture to the other.

ICDL-Brasil (ICDL-Brasil, s/d) is a binational cooperation project, whose aim is to make ICDL useful and usable for Brazilian children, in an attempt to encourage them to read and write more and better. A vast share of the Brazilian population is plagued by functional illiteracy. According to recent demographics (INAF, 2005), only 26\% of Brazilians between ages 15 and 64 are fully capable of reading and writing. The first step in the project is to assess how users immersed in a Brazilian context interact with the current ICDL interface. Among the tools we have used to this end is a qualitative method proposed by semiotic 
engineering: the communicability evaluation method, CEM (de Souza, 2005; Prates et al., 2000).

Unlike in most previous ICDL evaluations (e.g. Bilal, 2007; Druin, 2005; Hutchinson et al., 2005), our participants were not children, but adults. Although this may sound surprising, the role of adults in getting young children interested in reading has been shown to be crucially important (INAF, 2005). Hence, a critical requirement of ICDL-Brasil is that adults using ICDL with children feel comfortable and stimulated.

Our study has produced an in-depth perspective into some of the interpretive processes occurring in the context of typical interactions with ICDL. Our major findings are:

1. ICDL should provide increased support for multicultural navigation in order to increase the sense of safety and comfort of users as they move across different cultural settings.

2. As a multilingual site, ICDL should pay closer attention to the various linguistic materials in the system (e.g. the interface language and the various languages in which the books are written), in order to prevent serious interactive breakdowns.

3. ICDL design would probably benefit from separating linguistic and pragmatic issues in multicultural interaction, especially with respect to different forms of manipulating and reading books in Eastern and Western cultures.

In the following we briefly present the gist of semiotic engineering. Next, we introduce CEM. Then, we present our case study. Finally, we discuss our findings in view of related research and present our conclusions.

\section{Semiotic engineering: communicability and culture}

Semiotic engineering (de Souza, 2005) is a semiotic theory of HCI that views human-computer interaction as a particular case of metacommunication (communication of/about communication). Assuming that software is an intellectual artifact, the result of rational decisions and choices, and is designed to achieve certain purposes and effects in order to benefit and/or please its intended users, semiotic engineering stresses the fact that software requires appropriate interactive presentation and introduction. In accordance with this theory, the natural way of promoting good encounters and experiences with technology is to tell users, at interaction time, what they need to know in order to take the best out of it. But the way to do so can vary widely.

A key concept for semiotic engineering is communicability. Communicability is the distinctive quality of interactive computer-based systems that communicate efficiently and effectively to users their underlying design intent and interactive 
principles (Prates et al., 2000). As metacommunication artifacts, systems communicate a message that is elaborated by designers (senders) and interpreted by users (receivers). Together at interaction time as interlocutors of a communication process, designers and users negotiate meanings. And one of the keys to negotiating meanings is the designers' ability to communicate their intent (de Souza, 2005). The essence of this communication is the answer that a designer (or spokesperson for a design team) can give to three sets of questions:

1. Who are the users of the product I have designed? What do they want or need to do? In which preferred ways? And why?

2. What is this product I have designed? What can it do for its users? How?

3. What kinds of interactions, conversations, can or should users have with it? What range of effects can these conversations achieve? Are they consistent with who my users are, and with the needs and expectations that they have?

Put together, the answers to the above questions compose a top-level metacommunication message that is sent from designers to users, through the system's interface. Users unfold and receive this message, as they interact with the system. Thus, a system's interface is at once a message and an interlocutor, in a twofold communication process. Users interact with the message in order to get it, fully. Since this interaction is itself a process of exchanging messages, the interface must speak for the designers at interaction time - be "the designer's deputy", as proposed by the theory.

In order to illustrate metacommunication, we can use an instance from Google Brasil (http://www.google.com.br), focusing on the internationalization and localization strategies they use. In Figure 1 we see Google's interface for Brazilian users, which is very similar to that of North American users (same content, same interaction patterns, same sign structuring). To this internationalization strategy, designers have added localization ones (a separate URL, Portuguese translation). A single English language sign is preserved - a link to Google in North America. Search results are also localized: Brazilian domains are considered more relevant than non-Brazilian ones, even when users are searching for a term in English.
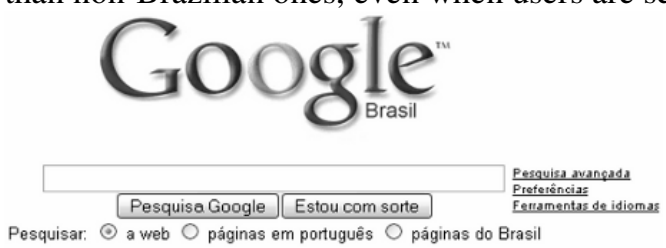

Soluç̋̈es de publicidade - Tuda sobre o Google - Google.com in English

ब2008 ooogle

Figure 1 Google Brasil homepage 
Now, what are the designers telling us (receivers of their message at interaction time)? The Google team communicates that users from certain cultures, like Brazil in this case, can benefit from an interface in Portuguese, with its own specific URL ('.br' suffix at the end of Google's generic 'www.google.com' locator). They also communicate their belief that Brazilian users prefer to access information in Brazilian Internet domains. However, if they wish to use the international Google version, they can reach it quite easily, by clicking on "Google.com in English".

This small glimpse into the reconstruction of the metacommunication message from Google Brasil helps us see what the designer-to-user communication process is and how it is achieved through the designer's deputy mediation at interaction time. It also helps us illustrate a potential communicative breakdown with the Google Brasil interface. When searching, Brazilian users may not realize that results from a search carried out in the Brazilian site do not come in the same order as results from the same search in the North American site. In other words, this localization effect is not clearly communicated. Only by comparison with searches in other Google sites does the user get the message.

Moreover, because the Google Brasil example so clearly directs us to cultural issues involved in the design of a very simple interface, it helps us understand why and how semiotic engineering is so tightly connected with culture. European semiotic theories (Eco, 1976) view communication as a cultural process in which we express ourselves through signs. Interpreting a message involves giving meaning to signs, signs that are most often established by virtue of cultural conventions. Thus, when we say that in semiotic engineering HCI is a particular case of metacommunication, we are actually implying that everything that constitutes an $\mathrm{HCI}$ issue is, in essence, a cultural issue.

Although the concept of culture is not explicitly defined in semiotic engineering, it is inexorably bound to this theory's basic ontology. Umberto Eco (1976), for instance, defines semiotics as "the logic of culture", and Clifford Geertz (1973) argues that the nature of culture is essentially semiotic. Culture, in this perspective, is a shared signification system that makes our behavior and the world around us meaningful; it shapes the way we interpret reality. So, metacommunication is a cultural process by which designers express a considerable extent of their world view.

\section{The Communicability Evaluation Method}

The Communicability Evaluation Method (CEM) (Prates et al., 2000) is a qualitative evaluation method developed to capture communicability problems, which are ultimately related to metacommunication issues. CEM privileges the reception of the designers' metacommunication, and produces not only an identification and explanation of problems, but also information for redesign. 
CEM introduces empirically-grounded pieces of knowledge in the collection of interpretive resources that designers can use to make sense of observed reality. Like other qualitative methods (Denzin and Lincoln, 2003), CEM emphasizes the diversity and richness of meanings involved both in users' and designers' interpretations of empirical reality. Each user's test session is unique and also bears many possible interpretations. Actually, the interpretation of several participants' results does not promote a consensual and convergent set of replicable parameters, but rather a set of empirical knowledge that stimulates the designers' critical and creative reflection about the metacommunication.

CEM is carried out in four steps: test preparation, tagging, interpretation and semiotic profiling. It involves observing users during interaction, analyzing these observations, exploring the application further, and finally explaining identified metacommunication problems, along with suggestions for redesigning the application.

The test preparation step is similar to typical user testing preparations, except that it requires that the evaluators elaborate an explicit description of the metacommunication message, and relevant test scenarios, with appropriate contextual cues for the analysis of metacommunication.

Tagging is the heart of CEM. Once user sessions have been recorded, evaluators watch the recorded movies and "put words on the users' mouths", in a kind of after-the-fact reconstruction of a verbal protocol. This is not a free text annotation of the recorded interaction, but a principled association of thirteen technically defined tags to those portions of the movies where evaluators detect communicability problems. For sake of illustration, among the communicability tags used in CEM, we have: "Where is it?"; "Where am I?"; "Oops!"; and "I can't do it this way.".

"Where is it?" is tagged in contexts where user knows what she is trying to do but cannot find an interface element that will tell the system to do it. She typically navigates through web pages or opens and closes dialogs, looking for that particular element.

"Where am I?" is tagged when the user is telling things to the system that would be appropriate in another context of communication, but not in the current one. Symptoms may include trying to select objects that are not active in the current context, trying to interact with signs that are output only, and so on.

“Oops!" is tagged when the user makes an instant mistake, and immediately tries to correct herself. A typical symptom of "Oops!" is to undo the faulty operation triggered by miscommunication.

Finally, "I can't do it this way." is tagged when, while trying to achieve a goal or sub-goal, the user engages in a several-step sequence of operations, but suddenly realizes that this is not the right thing to do. So, she abandons that long sequence, and takes a different path.

The interpretation step amounts to determining how successful the designers' communication is. Success is associated to the absence (or insignificant amount) of communicative breakdowns. Evidence from the tagging step helps evaluators 
decide on the quality of such communication. The following factors help the evaluators identify and understand communicability problems:

1. How often, and in which particular context, each type of tag appears;

2. The occurrence of tagging patterns;

3. Regular associations of tag types or sequences with problems in establishing communicative goals; and

4. When using additional evaluation methods, a correspondence between the locus of tag occurrence and that of problems indicated by the other methods. At the end of this step, evaluators should be able to tell when, where, how and why observed users were unable to: express what they meant; understand the system's expressions; choose the right way to communicate their intent; assigned the right meaning to what the system was communicating; or formulate a communicative intent altogether.

Semiotic profiling is the final step in CEM. The goal at this stage is to identify and explain problematic interaction design, and to inform redesign. They reconstruct the designer-to-user metacommunication message based on evidence provided by tests with users and on further exploration and inspection of the application

CEM thus achieves two important results. First, it gathers relevant evidence for redesign. Second, CEM expands the evaluators' and the designers' knowledge about HCI. For design, in particular, semiotic engineering explanations for communicative breakdowns can be the seed to more elaborate reasoning and decision-making when choosing between design alternatives, or when generating alternatives themselves. This is why, CEM is an epistemic tool. Its purpose is not to dictate solutions to a problem, but to support problem-solvers in naming and framing design problems, in generating solutions, and evaluating them ones against others (de Souza, 2005).

In the next section we show examples of CEM results when applied to ICDL.

\section{The ICDL Study}

One of the main practical goals of ICDL-Brasil (ICDL-Brasil, s/d) is to elaborate a culturally-adequate interface for ICDL, so that it can be productively used by Brazilian children and adult tutors. Thus we started the project with a communicability evaluation, looking for breakdowns related to cultural issues.

In the preparation phase we selected the cultural dimensions that would guide our study: language and pragmatics. Language, in this context, refers to the diversity of tongues, and pragmatics refers to the diversity of behavioral practices and attitudes involved in language use and in dealing with linguistic objects (Leech, 1983). There are several reasons for our choice. Firstly, the fact that 
languages encode (and thus reveal) culture; that is, they are primary signification systems (Geertz, 1973). Secondly, ICDL is a collection of instances of language use - many languages are spoken in and with ICDL (through books and interfaces). And finally, an important part of the ICDL mission is to foster "tolerance and respect for diverse cultures, languages, and ideas" (ICDL, s/d).

Next step, still in the preparation phase, we defined the participants' profile. Because of the leading role of adults in promoting children's literacy (INAF, 2005), we decided to start with a group of young adults. Participants were six students with experience in intercultural exchange programs, who could speak at least one foreign language. All of them had had previous experience with children (e.g. as teachers, baby sitters, and so on). The group was a bi-national one: three French and three Brazilian students. Note that there were no participants from the same culture as ICDL developers (North American). The last step in the preparation phase was a careful inspection of ICDL, from which we reconstructed the designers' metacommunication message and derived a test scenario.

At test sessions, all participants received the following assignment: "Suppose that you are a teacher or educator working with a group of 8-year olds. Because you loved your intercultural exchange experiences in the past, you want to light up their interest and curiosity about foreign cultures. Your goal is to search this website and choose a book to show them. You want to be original: you want to pick up a book in a language that they really don't understand, in this case in Persian or Farsi. This should trigger their imagination. It could be about music or poems."

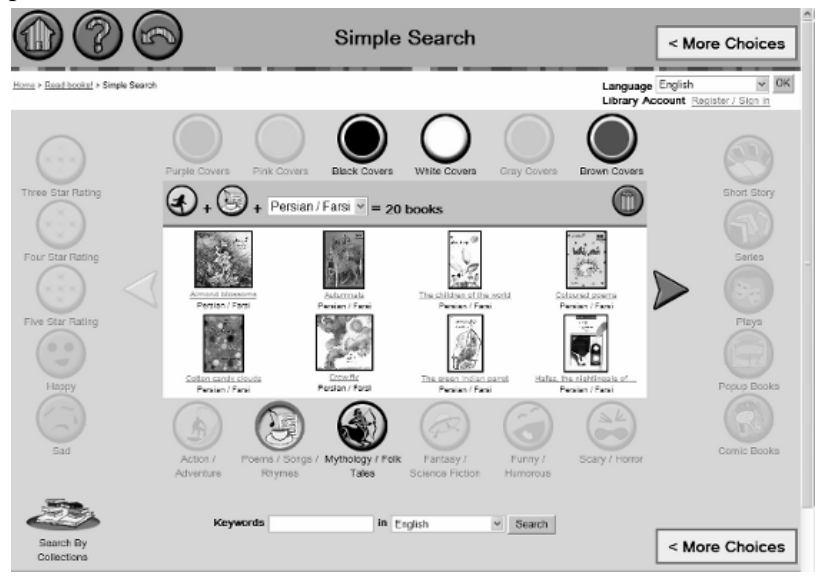

Figure 2 A simple search for books about poems / songs / rhymes in Persian/Farsi

The task was fairly open-ended, and explicitly addressed the multicultural character of ICDL. In Figure 2 we see a snapshot of a simple search returning the kinds of books that participants might choose. Using the simple search for the task is very convenient. The set of books to choose from can be obtained in only four 
steps: clicking on the appropriate age group; selecting the Persian/Farsi language; asking for "more choices"; and clicking on type "poems / songs / rhymes".

However, it took participants more than 8 minutes on average to finish the test. None of them searched for books in the way suggested in Figure 2, but all except one (who chose a book in Serbian) found an appropriate book.

Tagging was the next step and, below, we present the recurrent tags of our study.

Oops!: All participants except one were deeply confused by the language choice when searching a book (see Figure 3). They took "language" to mean book language, not interface language. Thus, when they set it to Persian/Farsi, they got into serious trouble. None of them could understand Persian/Farsi, so this was a mistake they had to correct. However, this "Oops!" was an expensive one, as will be seen below.

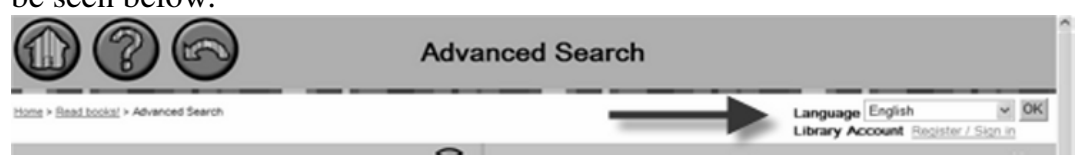

Figure 3 Choosing the interface language in ICDL

Where is it?: When ICDL users first access the website, all communication is in English, which is perfectly acceptable. Our tests provided evidence that when users inadvertently set the interface language to Persian/Farsi, they immediately tried to reset the interface language. They started looking for an interface element (a sign) to communicate they wanted to undo the faulty language setting, to set it back to the English default. A common pattern in most recorded sessions was to navigate back to the homepage (in English), hoping that this action would lead back to the default language interface. In other words, while receiving metacommunication, they took "homepage" to mean the same as "restart", which is a misinterpretation of the concept of homepage in web navigation that concomitantly achieves some procedure. Another pattern was trying to set the language back to the desired value. The problem is that participants had to know how to say "English" in Persian/Farsi. Since they didn't, interaction degraded into a lengthy quest for comprehensible communication.

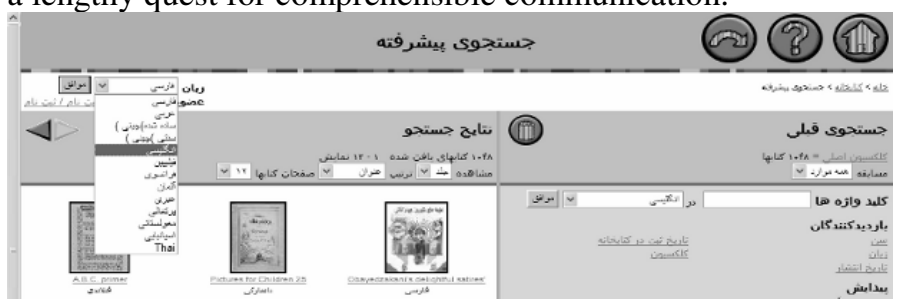

Figure 4 Setting the interface language back to English in Persian/Farsi

Where am I?: The inversion of navigation hints in the Persian/Farsi interface confused most participants. For example, advancing (going forward) and returning 
(going backward) were signified the other way around for Westerners. So, whenever they used arrows to mean "back/previous", they were actually telling the system to move "forward/next". The problem also appeared when the interface language was right for the user, but the desired action was to preview a book. When a Persian/Farsi book is previewed, the pages are displayed from right to left. This caused great confusion because it took some participants a while to realize why what they thought should be the book's first book page was an arbitrary page spatially located on the top-left corner of the overviewing area (see Figure 5).

I can't do it this way.: The location search is primarily a search by continent, and not by country as the link "books by country" suggests. Only when a particular continent is selected can the search be narrowed to a country (e.g. Asia and Middle East > Iran). This strategy cannot be combined with other criteria, such as content and age for instance. Thus, participants that chose this strategy could only meet their goal if they looked into the various books' metadata or browsed the books trying to decide whether books were appropriate or not. Moreover, a book "from a country" in ICDL is not necessarily written in this country's official language. Thus, participants who tried to use this kind of search either switched to another strategy after a while, or spent a considerable amount of time browsing books till they found one they liked.

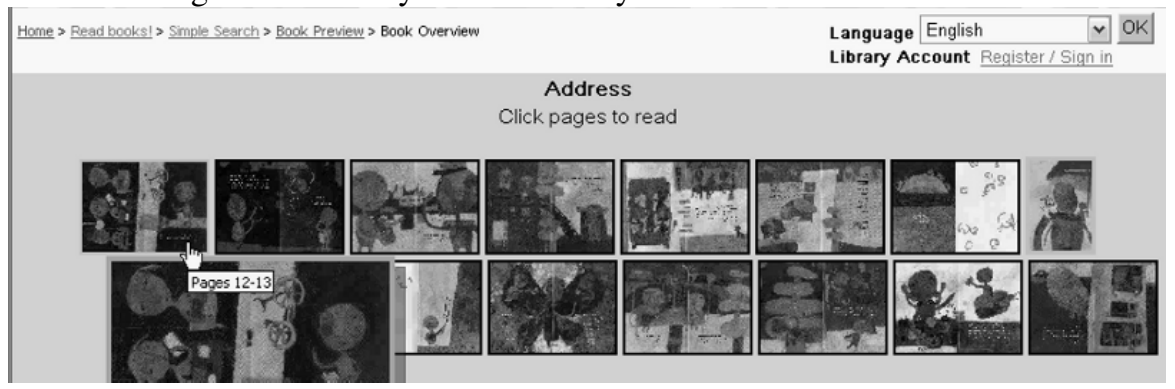

Figure 5 English interface overview page of a book in Persian/Farsi

After tagging, we interpreted results. Table II summarizes our main findings. The first column indicates a communicability problem. The second indicates whether we take it (" $\checkmark$ ") as a cultural problem related to language. And the third column indicates whether we take it as a cultural problem related to pragmatics.

Some problems are pragmatic, rather than linguistic, in a strict sense. For instance, a native speaker of French may not understand Finnish. Yet, if this person picks up a book in Finnish, the order of pages and direction of reading will be intuitive, even though the words can't be understood. Contrastively, if this person cannot speak Hebrew, and picks up a book in Hebrew, she may or may not realize that the direction of reading is reversed. Notice that, just as with Finnish, she doesn't understand the language. However, there is a sense of familiarity in manipulating books, identifying printed letters, and so on, that is much stronger with Finnish than Hebrew. This familiarity stems from practices and attitudes 
related to language use and dealing with linguistic objects regardless of how well one can understand one or another idiom.

Table 1 A summary of CEM results

\begin{tabular}{|l|c|c|}
\hline Communicability Problems & Language & Pragmatics \\
\hline $\begin{array}{l}\text { "Books by country" is an ambiguous expression (books by country } \\
\text { books by continent; a country's book may not be written in the } \\
\text { country's language). }\end{array}$ & $\checkmark$ & \\
\hline $\begin{array}{l}\text { Out-of-sync metadata translation may expose users to unexpected } \\
\text { changes of language during interaction (interface in Portuguese, } \\
\text { metadata in English). }\end{array}$ & $\checkmark$ & $\checkmark$ \\
\hline Users could not find how to reset the interface default language. & $\checkmark$ & $\checkmark$ \\
\hline $\begin{array}{l}\text { Users were confused with the order of reading / navigation when } \\
\text { exposed to Persian/Farsi interface and book previewing. }\end{array}$ & $\checkmark$ & $\checkmark$ \\
\hline $\begin{array}{l}\text { Users are confused by "interface / keyword / book" language } \\
\text { settings. }\end{array}$ & $\checkmark$ & \\
\hline $\begin{array}{l}\text { Multilingual representations and multilingual instances of books can } \\
\text { be easily confused (translated metadata } \neq \text { a translated version of the } \\
\text { book). }\end{array}$ & & $\checkmark$ \\
\hline
\end{tabular}

Other problems are strongly related to language, and only weakly related to pragmatics, like translation problems. For example, there was some confusion about books, themselves, and books metadata. The metadata may appear in two languages (e. g. English, the default, and French). When users see a book cover on screen labeled "Mushrooms in the rain", and later the same book cover is shown with the label "Champignons sous la pluie", they cannot tell whether these are two books, or the same book, whose title is translated into a foreign language.

Our interpretation of tagging led us to relevant insights in the semiotic profiling stage. We concluded that the main communicability issues in ICDL are three.

First, as a multicultural online environment par excellence, ICDL should provide increased support for multicultural navigation. Users should not lose sight of their native cultural markers when they move across different cultural settings. The presence of such markers (e.g. their native language, their homeland or starting point in the multicultural journey, etc.) could increase their sense of safety and comfort, and encourage them to take even wilder journeys in the ICDL globe. Our scenario, where an Eastern language and culture was chosen as a target for Western users, was deliberately chosen. It is in tune with the ICDL design vision. However, in the absence of cultural markers, users experienced serious problems with the interface.

Second, as a multilingual site, ICDL should separate different dimensions and classes of languages in its interface, and communicate differences very clearly. In the analyzed interface, all these languages were bundled together: the interface language, the original book language, the languages into which a book is translated, and the metadata language. 
Finally, decoupling linguistic from pragmatic issues in multicultural interaction may in fact benefit design. The problem with the direction of reading and book pagination is an important one. When reading a book, the user is supposed to understand the book language. Thus, within the scope of a visualized page, the direction of reading is of course affected by the particular graphical encoding adopted by that language. Hence, Persian/Farsi text can only be read right to left. However, book manipulation has to do with cultural practices that are languageseparable. Our inspection of ICDL has shown that the direction of reading in ICDL can be very confusing. Not only can users find the situation depicted in Figure 5, but speakers of languages encoded from right to left may even be more confused when they browse pages of books encoded from left to right - the meaning of arrows in pagination is difficult to figure out. Decoupling language from book manipulation allows designers to think of users' intuitions about book pagination, and design better browsing strategies for books written in languages unknown to the user.

\section{Discussion and Conclusions}

The use of CEM as an evaluation tool in the study of ICDL has yielded useful results. The need for cultural references to orient ICDL users as they move across cultural boundaries is in line with Barber and Badre's findings about the role of cultural markers in improving the usability of international websites (Bilal, 2007).

Likewise, much of the problems we have treated as linguistic have been dealt with in cross-cultural research by Bourges-Waldegg and Scrivener (1998), although as generalized meaning and representation problems. The authors concede, however, that "particular linguistic representations [...] are a design issue only if they become an obstacle to understanding." (p. 301). And this is precisely what CEM has allowed us to verify in the ICDL interface.

We also share many of their views. For example, it is our common belief that cross-cultural communication in natural contexts is probably less problematic than culturally-oriented HCI research may lead us to believe. Even coming from different cultures, people are naturally aware that signs may be interpreted differently by foreign interlocutors. This multicultural awareness can not only be sustained, but also increased, by culturally-informed HCI design.

As mentioned before, CEM is a qualitative method, whose aims is not to generate universal knowledge, predictive or prescriptive. CEM results are always contingent to a specific context, in our case the specific ICDL interface with which our participants interacted. However, CEM's epistemic nature allows results to become part and parcel of a design knowledge base. Thus, semiotic engineering explanations for communicative breakdowns can be the seed for more elaborate reasoning and decision-making when choosing between design alternatives, or generating new alternatives. Hence, we believe that the findings in 
this study are relevant pieces of knowledge that can be used to make sense of others multicultural environments,as shown next.

The first finding refers to the presence of native cultural markers - the native language, the homeland or the starting point on multicultural journeys. The Google Brasil example in section 2 discussed the presence of such marker, represented as a single link in a foreign language ("Google.com in English"). The second finding refers to a separation between interface language and various domain languages. Here again we can go back to the Google Brasil example and see that although Brazilian users may be happy to see the interface in Portuguese, they may not be happy to realize that search results are influenced by the assumption that items in Brazilian sites (in Portuguese, too) are more relevant to the user than items in other sites across the world. The problem stems from equating language and culture, whereas language is actually an element of culture (though possibly the most important one).

Finally, our third finding stresses the importance of treating linguistic and pragmatic issues as different focal points in the design process. The manipulation of linguistic objects is especially relevant, for example, in the design of online manga reading sites. Manga are Japanese cartoon-like stories that, in printed form, are read according to Japanese reading practices, in the opposite direction of Western cartoons. Various online reading sites for manga (e.g. http://www.readmanga.com) explore the possibilities of computer technology precisely to help Western readers follow the stories more comfortably, without the disorientation that results from reading manga in print. Unlike what happens in ICDL, manga online readers typically strive to help Western users by adopting a Western pagination and book manipulation standard (see figure 6).

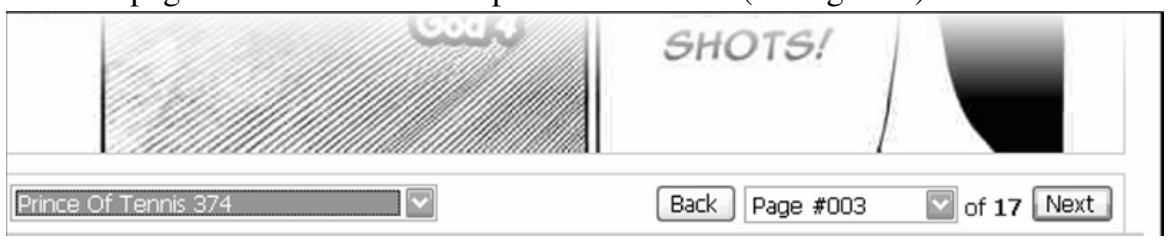

Figure 6 Interface of a manga viewer (http://www.mangavolume.com)

Based on these few examples, we consider that our results can contribute to support problem-solvers in naming and framing cultural design problems and in generating solutions for the design not only of ICDL but also of multicultural environments.

Acknowledgments This research is financially supported the National Council for Scientific and Technological Development (CNPq), Brazil, and is carried out in international cooperation with the ICDL research team at the University of Maryland, College Park. The authors would like to thank all the test participants. 


\section{References}

Aykin, N.: Usability and internationalization of information technology. Lawrence Erlbaum, Mahwah (2005).

Barber, W, and Badre, A.: Culturability: The merger of culture and usability. Proceedings of the 4th Conference on Human Factors \& the Web (1998). http://zing.ncsl.nist.gov/hfweb/att4/proceedings/barber/index.html. Accessed Jan 2007.

Bilal, D., and Bachir, I.: Children's interaction with cross-cultural and multilingual digital libraries: Understanding interface design representations. Information Processing and Management. 43: 47-64 (2007).

Bourges-Waldegg, P., and Scrivener SAR Meaning, the central issue in cross-cultural HCI design. Interacting with Computers. 9:287-309(1998).

Castells, M.: The Internet galaxy. Oxford University Press, Oxford (2001).

de Souza, C. S.: The Semiotic engineering of human-computer interaction. The MIT Press, Cambridge (2005).

Denzin, N. K., and Lincoln, Y. S.: The Sage Handbook of Qualitative Research. Sage, Thousand Oaks, California (2005).

Dray, S.: Designing for the rest of the world: A consultant's observations. Interactions.. 3(2), 1518 (1996).

Druin, A.: What children can teach us: developing digital libraries for children with children. Library Quarterly 75(1): 20-4 (2005).

Geertz, C.: The Interpretation of Cultures. Basic Books New York.

Eco, U.: A theory of semiotics. Indiana University Press, Bloomington (1976).

Hutchinson, H., Rose, A., Bederson, B., Weeks, A., and Druin, A.: The International Children's Digital Library: A case study in designing for a multi-lingual, multi-cultural, multigenerational audience. Information Technology and Libraries, 24(1): 4-12 (2005).

ICDL: The International Children's Digital Library. (s/d). http://www.icdlboorks.org Accessed Jan 2007.

ICDL-Brasil: Uma biblioteca internacional digital para crianças brasileiras. (s/d). http://www.icdl-br.inf.puc-rio.br Accessed Jan 2007.

INAF 5o Indicador Nacional de Alfabetismo Funcional: Um diagnóstico para a inclusão social pela educação. Instituto Paulo Montenegro, Ação Educativa, São Paulo (2005).

Kaplan, N., and Chisik, Y. Reading Alone Together: Creating Sociable Digital Library Books, In Proceedings of IDC 2005. New York: NY. ACM Press. pp. 88-94 (2005).

Leech, G. N.: Principles of Pragmatics. Longman, London (1983).

Marcus, A.: User interface design and culture. In N. Aykin (Ed.) Usability and internationalization of information technology. Mahwah: NJ. Lawrence Erlbaum. pp. 51-78 (2005).

Prates, R. O., de Souza, C. S., and Barbosa, S. D. J.: A method for evaluating the communicability of user interfaces, Interactions 7(1): 31-8 (2000).

Searle, J. R.: Speech acts: An essay in the philosophy of language. Cambridge University Press, Cambridge (1969). 